\title{
Does High Citrus Production Increase The Frequency of Consumption and Affect The Prevalence of Dental Erosion?
}

\author{
Ayșe Tuğba Ertürk Avunduk' (D), Esra Cengiz Yanardag' (D), Nazan Kocak Topbas² (1) \\ 'Department of Restorative Dentistry, Mersin University Faculty of Dentistry, Mersin, Turkey \\ 2Department of Oral and Maxillofacial Radiology, Mersin University Faculty of Dentistry, Mersin, Turkey
}

ORCID iDs of the authors: A.T.E.A. 0000-0002-7879-8I50; E.C.Y. 0000-0002-265I-2755; N.K.T. 0000-0002-3717-2098.

Cite this article as: Ertürk Avunduk AT, Cengiz Yanardag E, Kocak Topbas N. Does High Citrus Production Increase The Frequency of Consumption and Affect The Prevalence of Dental Erosion? Cyprus J Med Sci 2021; 6(I): 67-73.

\section{BACKGROUND/AIMS}

The authors have investigated the prevalence and reasons for erosive tooth wear, which has a complex and multifactorial etiology, in adolescents and adults in the eastern Mediterranean coastal city of Mersin, Turkey.

\section{MATERIAL and METHODS}

This cross-sectional, descriptive study was performed from June 2019 to February 2020 using a survey at the Faculty of Dentistry, Mersin University, Turkey. The questionnaire forms containing an informative section about the study were distributed to the participants. Data on general sociodemographic variables and risk factors were obtained. The survey consisted of 16 questions, including questions on demographic information, general health status, oral health status, and acidic dietary intake time; an oral examination was performed at the end of the survey. The data were statistically analyzed using the Student's t-test and one-way ANOVA.

\section{RESULTS}

There was a significant difference in the total erosion scores of the patients with respect to age $(p=.000)$, whereas no significant differences among the total erosion scores of the patients regarding gender were observed. The presence of erosive tooth wear was significant in terms of the frequency of consuming sour snacks and fruit juice.

\section{CONCLUSION}

Dental erosion was associated with both internal and external risk factors. Maxillary and mandibular anterior teeth had more erosive wear.

Keywords: Citrus consumption, dental erosion, epidemiology, prevalence, tooth surface loss

\section{INTRODUCTION}

Dental erosion is the irreversible progressive loss of dental hard tissues as a result of chemical acid or electrochemical mechanisms from a nonbacterial source. The erosive potential of erosive agents depends on chemical factors ( $\mathrm{pH}$, mineral content, and calcium-chelation properties) or biological factors (tooth anatomy, the relationship between soft tissue and teeth, saliva flow rate, and buffering capacity). Furthermore, behavioral factors, including eating and drinking habits, regular exercise resulting in dehydration and a decrease in salivary flow, excessive oral hygiene, and an unhealthy lifestyle, are predisposing factors for dental erosion (I).

The presence of acid solutions in the frequent and prolonged oral environment at values below the critical $\mathrm{pH}$ (5.5) causes the destruction of the enamel tissue due to erosion. Acidic solutions may be of internal or external origin; nevertheless, the frequent consumption of acids is not alone responsible for dental erosion (2). There can be predisposing factors, such as acquired dental pellicle, weakened tooth structure, and reduced buffering capacity, that may influence a person's susceptibility to erosive tooth wear. In terms of long-term dental health, erosive tooth wear has been becoming progressively important according to epidemiological studies, with some evidence showing that the prevalence of dental erosion has increased (3). In the previous studies related to the prevalence of erosion, different results have been reported for deciduous and permanent dentition (4). It was stated that different erosion rates were seen in 
studies on the prevalence of erosion owing to the differences in scoring systems, sample selection, and research techniques. Therefore, it can be complicated to evaluate the results of the prevalence studies.

There is no diagnostic device that measures the amount of wear for clinical detection of dental erosion; therefore, clinical appearance is the most important symptom in the diagnosis of erosive lesions. Generally, erosion begins on the tooth surface, with a flat, bright, and shallow concave shape. Then, it displays a stepped surface appearance as long as the causative agent is not eliminated (3). Different index systems have been used by researchers from the past to the present to evaluate and score dental wear. Eccles (5) has broadly classified lesions as baseline, early, small, and severe without definite criterion definitions, thus allowing a broad interpretation. Xhonga and Valdmanis (6) measured erosion lesions with a periodontal probe and made the scoring in four levels: none, small ( $<2 \mathrm{~mm}$ ), medium (up to 3 $\mathrm{mm}$ ), and heavy (>3 mm). Tooth Wear Index is a numerical scale used to measure tooth tissue loss in millimeters and to determine the presence of secondary dentin (6). Basic Erosive Wear Examination was introduced at a conference in Basel, Switzerland in 2007, and it provides a simple scoring system that can be used with the diagnostic criteria of all available indices (7). The index designed by Lussi et al. (8) records the severity of erosion and is a more detailed modification of the index designed by Eccles and Jenkins (5).

There are many studies on the prevalence of dental erosion (9). However, the literature review revealed no data regarding the dental erosion status of individuals who lived in the eastern Mediterranean coastal city of Mersin, which is one of the most important cities in terms of citrus production in Turkey. The aim of this study was to determine the status of dental erosion in adolescent and adult individuals owing to the consumption of citrus products in Mersin. The null hypotheses were as follows:

I. There is no difference among the total erosion scores of the participants according to the age groups.

2. There is no difference in the prevalence of dental erosion between adolescents and young adults in terms of the importance of the research area with high citrus production.

\section{MATERIAL and METHODS}

This cross-sectional study was conducted from June 2019 to February 2020 using a survey. The protocol of this study was approved by the ethics committee of the Mersin University (Turkey) and was conducted in accordance with the most recent guidelines of the Declaration of Helsinki. The study was conducted following the strengthening the reporting of observational studies in epidemiology (STROBE) guidelines (I0). The sample size was

\section{Main Points:}

- Dental erosion is an irreversible progressive loss of dental hard tissues.

- Dental erosion can be a public health concern because of its high prevalence in population research.

- The scoring methodology appears precious for assessing erosion in populations. calculated on the basis of the total target population for adolescents and adults (openepi.com/SampleSize) aged $>16$ years who were living in Mersin. The total number of adolescents and adults and a precision level of $\pm 5 \%$ for the $80 \%$ confidence interval were used to calculate the number of participants to be included. As a result of the sampling calculation, it was determined that a minimum of 106 participants should randomly be selected.

\section{Ethical Approval}

The study protocol was approved by the clinical research ethics committee of the Mersin University (approval number: 2019/215-22.05.2019). The study conformed with the most recent guidelines of the Declaration of Helsinki and was performed according to the guidelines of the STROBE checklist. Before participation, all patients or their parents/legal guardians received oral and written study information and signed a written consent form.

Routine intraoral and radiological examinations of the 1,957 patients were performed in the Department of Oral and Maxillofacial Radiology, Mersin University. A total of 489 of these patients were diagnosed with noncaries cervical lesions (NCCLs) and were directed to the Department of Restorative Dentistry. Intraoral and radiological examinations were repeated, and their anamnesis was detailed in this department. Subsequently, a questionnaire was applied to the participants who had dental erosion. Eligibility criteria were as follows: those with age $>16$ years, those who gave consent to participate in the study, those who completely answered the questionnaire (Figure I). Exclusion criteria consisted of cases of abrasion, attrition, and abfraction (which are NCCLs but were not included) and those with age $<16$ years. A total of 163 patients $(n=163)$ participated in this survey.

The research questions were modified using a survey prepared by Chu et al. (II) and Pineda et al. (I2). A pilot testing on 20 patients was conducted, and no adjustments to the questionnaire were made. The questionnaire used in this study consisted of three sections and 16 questions. After a part explaining the purpose of the questionnaire, the first section was about obtaining the demographic data (sex, age, profession) of the participants. The second part of the questionnaire consisted of health problems and medications, tooth brushing habits, the use of fluoride, and dietary habits (Table I). At the end of the questionnaire, a clinical examination was performed for the participants who

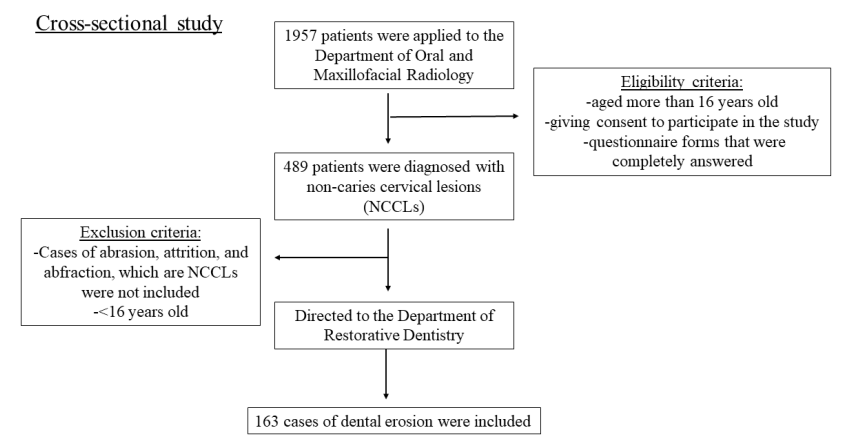

FIGURE I. Flow chart for the study 
gave permission. One standardized examiner (A.T.E.A.) who was trained to identify erosive lesions performed the clinical examinations. The participants were seated under an artificial source of light and assessed with a dental mirror. The examiner dried the teeth with gauze before the clinical examination. The surfaces (occlusal/incisal, buccal, and lingual) of the teeth except those of the third molars were evaluated for each individual in this study. The status and prevalence of tooth erosion were scored using the modified criteria of Lussi (8).

\section{Statistical Analysis}

All data were stored in specially arranged computer files. The statistical analyses were performed using the Statistical Package for the Social Sciences, version 25.0 (IBM SPSS Corp.; Armonk, NY, USA). The Student's t-test was used to compare the total erosion scores of the patients according to sex, systemic disease, drug usage, consumption of sports drinks, and the patients' perception of dental problems. One-way analysis of variance test was used to compare the total erosion scores of the patients according to the age, the type of systemic disease, the number of daily brushing of teeth, the usage of fluoride toothpaste and auxiliary substances while brushing the teeth, and the consumption of sour snacks, fruit juice, and acidic beverages.

\section{RESULTS}

A total of $59.5 \%$ of 163 patients were female, and $40.5 \%$ were male. It was observed that $25.2 \%$ of the participants were aged between $16-25$ years, 38\% were aged between $26-40$ years, and $36.8 \%$ were aged $>40$ years. There were no differences in the total erosion scores of the patients according to sex, whereas there were significant differences according to age groups $(p=.001)$. The participants aged $>40$ years had significantly higher erosion scores than those in the $16-25$ and $25-40$ age groups (Table 2). The comparison of the total erosion scores of the patients in terms of systemic disease and drug usage is summarized in Table 3. There was no significant difference according to the type of systemic diseases ( $p>$.05). The scores of patients with systemic disease were significantly higher than those of patients without systemic disease. There were significant differences among the total erosion scores according to drug usage $(p=.002)$.

Table 4 shows that the total erosion scores of the patients did not differ according to the number of daily brushing of teeth and the use of fluoride toothpaste, auxiliaries, and mouthwashes ( $p>$.05). On the other hand, there was a significant difference in the number of patients according to the consumption of sour snacks ( $p=.0 \mathrm{l})$. The patients who responded "always" to the question about sour snacks consumption were significant-

TABLE I. The questionnaire used in the study

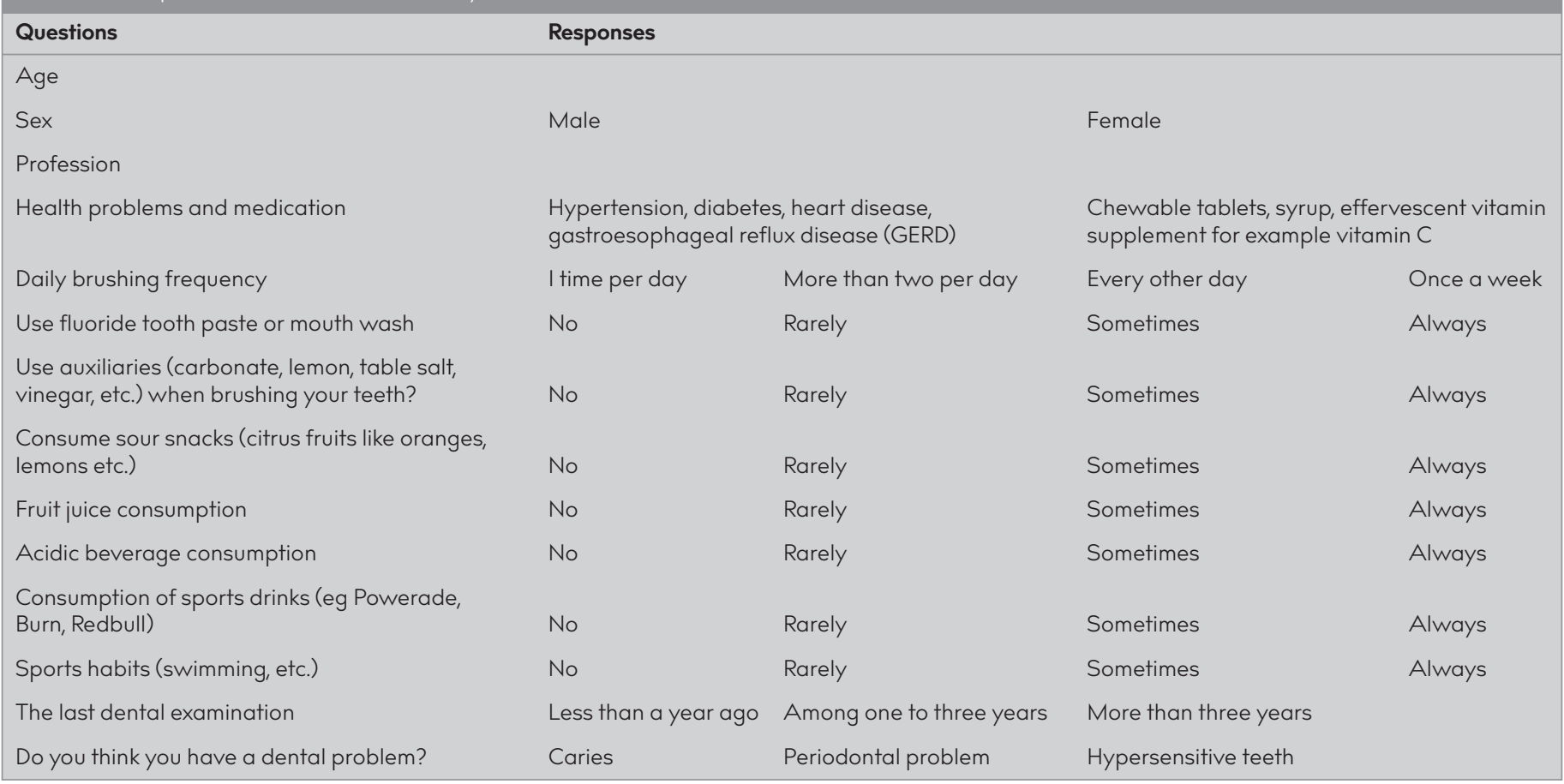

TABLE 2. Scoring of tooth erosion ${ }^{8}$

\begin{tabular}{|lll|}
\hline Grade & Anterior Teeth & Posterior Teeth \\
\hline 0 & No erosion & No erosion \\
\hline & $\begin{array}{l}\text { Loss of surface enamel, dentine not involved } \\
\text { Enamel erosion on cusp tips ("cupping" with dentine not obviously involved) or erosion of } \\
\text { fissures that cannot be attributed to attrition. }\end{array}$ \\
3 & $\begin{array}{l}\text { Erosion extending into dentine } \\
\text { Severe dentine erosion (in at least } 4 \text { anterior } \\
\text { teeth) and/or the pulp chamber visible }\end{array}$ & $\begin{array}{l}\text { Erosion extending into dentine that cannot be attributed to attrition } \\
\text { Eronding well into dentine and close to the pulp }\end{array}$ \\
\hline
\end{tabular}


ly higher than those who responded "sometimes." It was observed that the total erosion scores of the patients showed a significant difference in terms of fruit juice drinking ( $p=.03)$. The patients who consumed fruit juice "always" were significantly higher than those who consumed "sometimes." The consumption of carbonated and sports drinks and the habit of performing sports activities did not affect the erosion scores ( $p>05$ ).

The total erosion scores of the patients who had their last dental examination dates differed and having dental problems or tooth hypersensitivity did not show a significant difference $(p>.05)$. It was determined that there was a significant difference in terms of the patient's periodontal problem $(p=.01)$. Total erosion scores of patients who had periodontal problems were higher than the scores of those who did not have (Table 4).

A total of 4,564 dental examinations were performed by examining 28 different teeth of 163 patients. A total of $78.92 \%$ of the teeth $(n=3,602)$ had no erosion score, $17.62 \%(n=804)$ had one, $3.13 \%(n=143)$ had two, and $0.33 \%(n=15)$ had three. Maxillary and mandibular anterior teeth had more erosive wear, whereas the mandibular posterior teeth were the least commonly affected (Table 5). In a large proportion of the patients, the erosion scores of the teeth in the first, third, fourth, and sixth sextants were zero. However, it was observed that the teeth in the second and fifth sextants had intense signs of erosion.

\begin{tabular}{|c|c|c|c|c|}
\hline & $N$ & Mean & Sd & $\mathrm{p}$ \\
\hline \multicolumn{5}{|l|}{ Age } \\
\hline $16-25$ & 41 & 5,80 & 4,80 & \multirow[t]{3}{*}{$\mathrm{p}^{\mathrm{a}}=0,00 \mathrm{I}^{*}$} \\
\hline $25-40$ & 62 & 5,63 & 3,47 & \\
\hline$>40$ & 60 & 9,13 & 6,24 & \\
\hline \multicolumn{5}{|l|}{ Sex } \\
\hline Female & 97 & 6,81 & 4,92 & \multirow[t]{2}{*}{$p^{b}=0,66$} \\
\hline Male & 66 & 7,18 & 5,64 & \\
\hline \multicolumn{5}{|c|}{ Type of Systemic Disease } \\
\hline No Systemic Disease & 91 & 6,22 & 4,44 & \multirow{6}{*}{$p^{a}=0,062$} \\
\hline Hypertension & 9 & 11,33 & 9,04 & \\
\hline Diabetes & 4 & 9,25 & 8,22 & \\
\hline Heart disease & 8 & 9,00 & 9,74 & \\
\hline GERD & 27 & 6,70 & 3,35 & \\
\hline Other & 24 & 7,38 & 4,82 & \\
\hline \multicolumn{5}{|l|}{ Systemic Disease } \\
\hline No & 91 & 6,22 & 4,44 & \multirow[t]{2}{*}{$p^{b}=0,040^{*}$} \\
\hline Yes & 72 & 7,90 & 5,95 & \\
\hline \multicolumn{5}{|l|}{ Drug Use } \\
\hline No & 119 & 6,20 & 4,36 & \multirow[t]{2}{*}{$p^{b}=0,002^{*}$} \\
\hline Yes & 44 & 9,02 & 6,66 & \\
\hline $\begin{array}{l}\text { ap values are based o } \\
\text { b } p \text { values are based o } \\
{ }^{*} p<0,05 \text { is significant, }\end{array}$ & $\begin{array}{l}\text { y AN } \\
\text { lent } \\
\text { rd de }\end{array}$ & $\begin{array}{l}\text { A test } \\
\text { it } \\
\text { tion }\end{array}$ & & \\
\hline
\end{tabular}

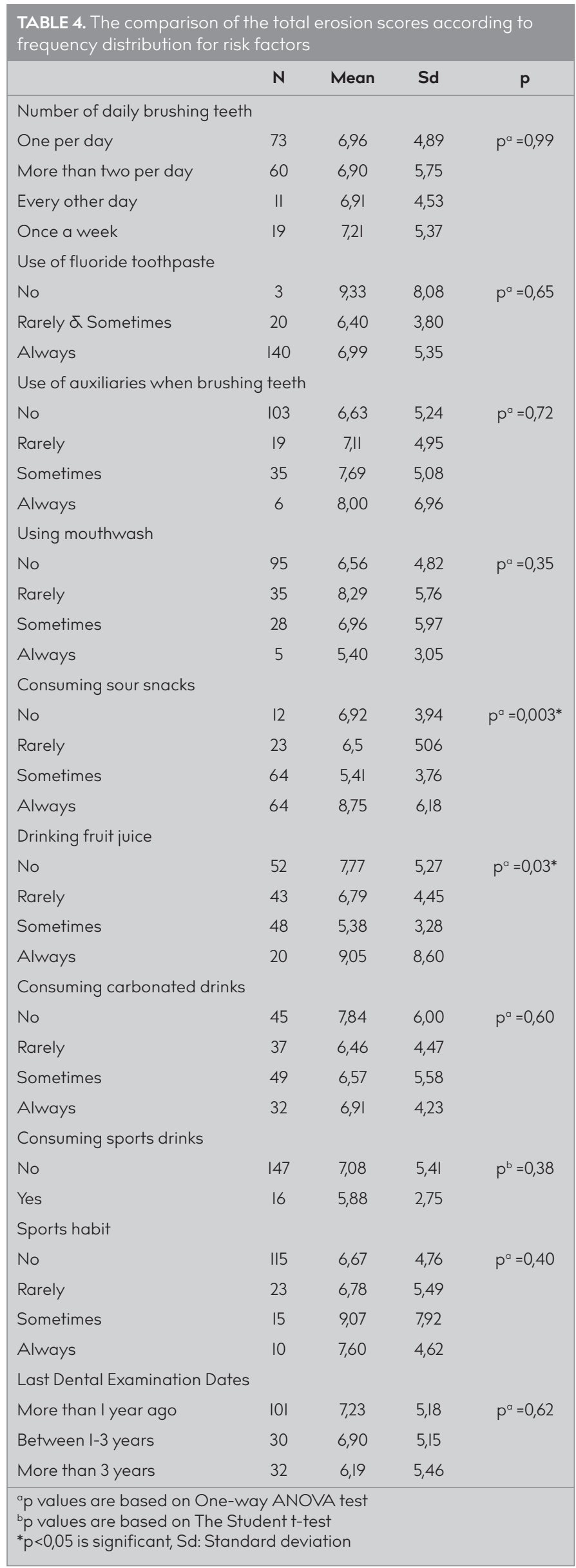




\begin{tabular}{|lccccc|}
\hline \multicolumn{5}{|c|}{ TABLE 5. Distribution of dental erosion degrees by mouth region } \\
\cline { 2 - 6 } Sextant & $\mathbf{0}$ & $\mathbf{1}$ & $\mathbf{2}$ & $\mathbf{3}$ & Total \\
\hline $1(17-14)$ & $617(94,6)$ & $26(4,0)$ & $9(1,4)$ & - & $652(100,0)$ \\
$2(13-23)$ & $577(59,0)$ & $322(32,9)$ & $69(7,1)$ & $10(1,0)$ & $978(100,0)$ \\
$3(24-27)$ & $624(95,7)$ & $20(3,1$ & $6(0,9)$ & $2(0,3)$ & $652(100,0)$ \\
$4(37-34)$ & $564(86,5)$ & $70(10,7)$ & $18(2,8)$ & - & $652(100,0)$ \\
$5(33-43)$ & $667(68,2)$ & $287(29,3)$ & $23(2,4)$ & $1(0,1)$ & $978(100,0)$ \\
$6(44-47)$ & $553(84,8)$ & $79(12,1)$ & $18(2,8)$ & $2(0,3)$ & $652(100,0)$ \\
\hline & & & & & \\
\hline
\end{tabular}

\section{DISCUSSION}

Nowadays, with the enhancement of consumption rates of acidic beverages, the percentage of dental erosion is increasing (I3). The main dietary acids for erosion are citric, phosphoric, malic, and tartaric acids. Fruits such as orange and lemon and vegetables mainly contain citric acid, which is followed by malic acid. In addition, citric acid is being added to many of the commercially produced products. Among the many agricultural products grown in Turkey, the production of citrus species is also playing an important role, and when the provinces that export most of the citrus fruits are examined, the Mersin region is in the first place. Thus, the purpose of this study was to determine the status of dental erosion in adolescent and adult individuals in Mersin.

It is recognized that epidemiological prevalence studies did not use an ideal index for clinical staging and monitoring of dental erosion and that there is no simple assessment system meeting all the needs of both clinicians and investigators (6). There are different dental index systems that vary according to the evalvation type, scoring system, and tooth selection (I4). This study was aimed to determine the severity of dental erosion and to identify whether the lesion involved enamel or enamel and dentine. We considered that having this information clinically could be critical for treatment decisions and important for prevalence studies. The modified criteria of the Lussi index, which evaluates the teeth separately in the anteroposterior areas according to the wear severity of the hard tooth tissue and has been used widely by European researchers ( 6 ) to score the facial, lingual, and occlusal surfaces of all teeth except those of the third molar, was preferred in this study.

According to the findings of this study, a significant difference was observed in total erosion scores with respect to the age of the participants. Therefore, the first hypothesis of this study, which suggested, "there is no difference among the total erosion scores of the participants according to the age groups," is rejected. Similar to this study, Vered et al. (15) reported a significant difference between the mean total erosion scores of five different age groups (15-18, 25-28, 35-38, 45-48, and 55-60 years). Regarding sex, there was no significant difference between male and female participants in this study (Table 3). This finding is in accordance with the previous studies conducted by Chu et al. (II) and Luciano et al. (16) who reported that no significant differences were observed between male and female individuals about the prevalence of dental erosion.

The prevalence of erosion has been reported to be $11 \%-100 \%$ in adolescents and $4 \%-82 \%$ in adults (I). In this study, the preva- lence of erosive tooth wear was 33.3\% (163 participants), and the total erosion scores of the participants aged $>40$ years were significantly higher than the scores of those aged 16-25 and 26-40 years (Table 3 ). Therefore, the second hypothesis of this study, which suggested, "There is no difference in the prevalence of dental erosion between adolescents and young adults in terms of the importance of the research area with high citrus production," is rejected. Similar to the findings of this study, Pineda et al. (12) reported that the prevalence of erosive wear was $31.7 \%$ in a sample of adolescents aged $14-19$ years, and Mathew et al. (17) stated that the prevalence of erosive wear was $36.5 \%$ in a population of individuals aged $18-28$ years. Kitasako et al. (18) evaluated the prevalence of erosion on all tooth surfaces of adults aged 15-89 years in Tokyo, Japan, and reported that the prevalence in the group who had erosion was $26.1 \%$. In this study, the prevalence of erosive tooth wear limited to enamel was found to be $17.6 \%$ ( 804 teeth) overall in 4,564 teeth, and the prevalence of erosive wear with exposed dentin was found to be 3.5\% (158 teeth). Our findings are in consonance with those of the previous study conducted by McGuire et al. (19) in the United States who reported that the prevalence of erosive wear with exposed dentin was $5.5 \%$. With the progression of age, changes in saliva structure (decreased buffering capacity, hyposalivation, and $\mathrm{pH}$ ) owing to factors such as systemic disease and eating or drinking habits may play a role in the exacerbation of erosive lesions. Nevertheless, it is thought that physiological or pathological gingival recessions that occur depending on age also increase the predisposition to erosion.

The presence of exogenous and endogenous acids in the diet has proven to play a role in the initiation and progression of dental erosion. Erosive tooth wear occurs more common in anorexia nervosa, bulimia, nervous vomiting, alcohol usage, and pregnancy cases (20). In addition, the passage of stomach contents into the esophagus without difficulty is defined as gastroesophageal reflux disease (GERD). The relationship between erosion and GERD has been shown in many studies, and erosive wear are frequently encountered on the palatinal and lingual surfaces of the teeth (2I). According to the findings of this study, the scores of the patients with systemic diseases were significantly higher than the scores of the patients without, and there were significant differences in the total erosion scores with respect to the presence of systemic diseases and drug usage. A total of 27 of the participants (19\%) had GERD in this study, and this was shown as the reason for the difference (Table 2). This is in agreement with the findings reported by Picos et al. (22).

The frequency distribution for risk factors of this study is summarized in Table 4. There was a significant association between the presence of erosive tooth wear and the frequency of consuming citruses such as orange, lemon, or other and fruit juice $(p<.05)$. The excessive consumption of sour snacks due to the high production in the region of the study may be the reason for this difference. On the other hand, there was no significant difference between the other risk factors and participants' erosion tooth wear. As a reason for this difference, it is thought that the consumption of such products increases in the location of this study owing to the high production. It has been reported that excessive and frequent consumption of citrus fruits due to their bioactive components, such as citric acid, ascorbic acid, and polyphenol, damages the hard tissues of the teeth (23). Jarkander et al. (24) 
investigated the prevalence and risk factors of dental erosion on adolescents in Stockholm and reported that soft drink consumption and the consumption of juice or sports drinks were significantly correlated with clinically diagnosed erosive wear. However, not in accordance with the results of this study, Luciano et al. (16)] reported that no significant difference was found between the citrus fruit and juice consumption and dental erosion risk in individuals aged between 12-30 years in the Brazilian city. On the other hand, in this study, it was determined that the total erosion scores of the participants who had periodontal problems were higher than those of patients who did not have periodontal problems (Table 4). It is known that the exposure of tooth crown and root surfaces due to the periodontal problems increases the frequency of dental erosion clinically (25).

Although dental erosion-related wear occurs on all surfaces of the tooth, it is reported that it often occurs on the palatal region of the maxillary anterior teeth and the occlusal surfaces of the lower first molar (26). Consistent with the literature, the second and fifth sextants had more erosive wear, whereas the mandibular posterior teeth were the least commonly affected (Table 5). The reason why more erosive wear on the anterior teeth was seen was that some participants expressed that they especially ate the lemon by biting it. However, in this study, in a large proportion of the participants, the erosion scores of the teeth in the first, third, fourth, and sixth sextants were zero. These results are not similar to those of the previous study conducted by Pineda et al. (12) and Bardolia et al. (27) who stated that the most affected regions were the occlusal surfaces of mandibular first molars. Most of the participants stated that they usually ate lemon by biting, which may be the reason for the excessive wear of the anterior teeth (caused by erosion) found in this study. In our study, it was observed that erosive tooth wear was more common in individuals aged $>40$ years, as stated before. Differences between anterior and posterior teeth depending on age may be explained by variations in dental restoration situations (crowns, onlays, inlays) or intraoral location because posterior teeth are more often missing or have full-coverage restorations than anterior teeth. The number of teeth remaining in the mouth could also be responsible for erosive tooth wear. In agreement with this result, Vargas-Ferreira et al. (28) and Gurgel et al. (29) reported the buccal surface of the maxillary central incisors as the most affected erosive wear site. On the other hand, Auad et al. (30) observed that erosive wear mostly occurs on the palatal surface of anterior teeth. The discrepancy between previous findings and these results may be explained by the evaluation type, specific population properties, different scoring systems, tooth selection, or diversity of the diagnostic criteria.

Cross-sectional studies provide limited data in a short period and at a one-time point. These studies are generally used to determine the prevalence of a disease in a population, but there is no recall and control over. The first limitation of this study is that the total erosion scores of the patients were compared according to the consumption frequency of carbonated drinks, sour snacks, or fruit juice. Therefore, further clinical studies should be designed to investigate the properties of saliva, such as flow and buffering capacities. The second limitation is the determination of total erosion tooth wear using one scoring system. Using different dental indices together will contribute to the sensitivity and specificity of the studies.
To manage dental erosion, it is important to diagnose the lesions at an early stage, identify possible etiology, and have knowledge regarding the protective and risk factors and their interactions. Clinicians should make recommendations to the patients considering the etiological factors for oral care and consider the individual needs and motivation of the patients to provide their oral health.

On the basis of the findings of this study and within the limitations, it seems appropriate to conclude that individuals aged $>40$ years have a higher risk in terms of the total erosion scores. The prevalence of erosive tooth wear may be associated with age, systemic disease, drug use, and consumption of sour snacks and fruit juice. Erosive tooth wear was observed on mostly maxillary and mandibular anterior teeth, whereas total erosion scores were lowest in the mandibular posterior teeth.

Ethics Committee Approval: Ethics committee approval was received for this study from the ethics committee of Mersin University (approval no: 2019/215-22.05.2019).

Informed Consent: Written informed consent was obtained from patients who participated in this study.

Peer-review: Externally peer-reviewed.

Author contributions: Concept - A.T.E.A., E.C.Y; Design - A.T.E.A., E.C.Y.; Supervision - A.T.E.A., E.C.Y.; Resource - A.T.E.A., E.C.Y.; Materials A.T.E.A.; Data Collection and/or Processing - A.T.E.A., N.K.T.; Analysis and/or Interpretation - A.T.E.A., N.K.T.; Literature Search - A.T.E.A.; Writing - A.T.E.A., E.C.Y.; Critical Reviews - A.T.E.A., E.C.Y.

Conflict of Interest: Authors have no conflicts of interest to declare.

Financial Disclosure: The authors declared that this study has received no financial support.

\section{REFERENCES}

I. Lussi A, Jaeggi T, Zero D. The role of diet in the aetiology of dental erosion. Caries Res 2004; 38(Suppl. I): 34-44. [Crossref]

2. Ganss C, Lussi A. Current erosion indices: flawed or valid? Clin Oral Investig 2008; 12(Suppl I): SI-S3. [Crossref]

3. Lussi A, Carvalho TS. Erosive tooth wear: a multifactorial condition of growing concern and increasing knowledge. Erosive Tooth Wear. Monogr Oral Sci 20l4; 25: I-I5. [Crossref]

4. Lussi A, Schlüter N, Rakhmatullina E, Ganss C. Dental erosion-an overview with emphasis on chemical and histopathological aspects. Caries Res 2011; 45(Suppl. I): 2-12. [Crossref]

5. Eccles J. Dental erosion of nonindustrial origin. A clinical survey and classification. J Prosthet Dent 1979; 42(6): 649-53. [Crossref]

6. Bardsley PF. The evolution of tooth wear indices. Clin Oral Investig 2008; 12(I): 15-9. [Crossref]

7. Bartlett D, Ganss C, Lussi A. Basic Erosive Wear Examination (BEWE): a new scoring system for scientific and clinical needs. Clin Oral Investig 2008; I2(I): 65-8. [Crossref]

8. Holbrook W, Árnadóttir I, Hlöðversson S, Arnarsdóttir E, Jónsson $\mathrm{S}$, Saemundsson SR. The Basic Erosive Wear Examination (BEWE) applied retrospectively to two studies. Clin Oral Investig 20I4; 18(6): 1625-9. [Crossref]

9. Li J, Fan W, Lu Y, Xuan D, Liu Z, Huang S, et al. A survey on the prevalence and risk indicators of erosive tooth wear among adolescents in Guangzhou, South China. J Oral Rehabil 2019; 46(6): 493-502. [Crossref]

10. Von Elm E, Altman DG, Egger M, Pocock SJ, Gøtzsche PC, Vandenbroucke JP. The Strengthening the Reporting of Observational 
Studies in Epidemiology (STROBE) statement: guidelines for reporting observational studies. Ann Intern Med 2007; 147(8): 573-7. [Crossref]

II. Chu $\mathrm{CH}, \mathrm{Ng}$ A, Chau A, Lo E. Dental erosion and caries status of chinese university students. Oral Hlth Prev Dent 2015; 13(3): 23744.

12. Pineda ÁEG-A, Borges-Yáñez SA, Lussi A, Irigoyen-Camacho ME, Medina FA. Prevalence of erosive tooth wear and associated factors in a group of Mexican adolescents. J Am Dent Assoc 2016; 147(2): 92-7. [Crossref]

13. Loke C, Lee J, Sander S, Mei L, Farella M. Factors affecting intra-oral pH-a review. J Oral Rehabil 2016; 43(I0): 778-85. [Crossref]

14. Wang $X$, Lussi A. Assessment and management of dental erosion. Dent Clin 20I0; 54(3): 565-78. [Crossref]

15. Vered Y, Lussi A, Zini A, Gleitman J, Sgan-Cohen H. Dental erosive wear assessment among adolescents and adults utilizing the basic erosive wear examination (BEWE) scoring system. Clin Oral Investig 2014; I8(8): 1985-90. [Crossref]

16. Luciano LCO, Ferreira MC, Paschoal MA. Prevalence and factors associated with dental erosion in individuals aged $12-30$ years in a northeastern Brazilian city. Clin Cosmet Investig Dent 2017; 9: 85-91. [Crossref]

17. Mathew T, Casamassimo PS, Hayes JR. Relationship between sports drinks and dental erosion in 304 university athletes in Columbus, Ohio, USA. Caries Res 2002; 36(4): 28l-7. [Crossref]

18. Kitasako Y, Sasaki Y, Takagaki T, Sadr A, Tagami J. Erosive Tooth Wear Among Different Tooth Types and Surfaces in Japanese Adults 15 to 89 Years Old. Oral HIth Prev Dent 2017; 15(4): 357-64.

19. McGuire J, Szabo A, Jackson S, Bradley TG, Okunseri C. Erosive tooth wear among children in the United States: relationship to race/ethnicity and obesity. Int J Paediatr Dent 2009; 19(2): 91-8. [Crossref]
20. Johansson AK, Norring C, Unell L, Johansson A. Eating disorders and oral health: a matched case-control study. Eur J Oral Sci 20I2; 120(I): 6I-8. [Crossref]

21. Pace F, Pallotta S, Tonini M, Vakil N, Bianchi Porro G. Systematic review: gastro-oesophageal reflux disease and dental lesions. Aliment Pharm Ther 2008; 27(12): I179-86. [Crossref]

22. Picos A, Badea ME, Dumitrascu DL. Dental erosion in gastro-esophageal reflux disease. A systematic review. Clujul Med 2018; 91(4): 387. [Crossref]

23. Bassiouny M. Clinical observations of dental erosion associated with citrus diet and intake methods. Gen Dent 2014; 62(I): 49-55.

24. Jarkander MS, Grindefjord M, Carlstedt K. Dental erosion, prevalence and risk factors among a group of adolescents in Stockholm County. Eur Arch Paediatr Dent 2018; 19(I): 23-31. [Crossref]

25. Chrysanthakopoulos NA. Gingival recession: prevalence and risk indicators among young greek adults. J Clin Exp Dent 2014; 6(3): e243. [Crossref]

26. Johansson A-K, Omar R, Carlsson GE, Johansson A. Dental erosion and its growing importance in clinical practice: from past to present. Int J Dent 2012; 2012: I-17. [Crossref]

27. Bardolia P, Burnside G, Ashcroft A, et al. Prevalence and risk indicators of erosion in thirteen-to fourteen-year-olds on the Isle of Man. Caries Res 2010; 44(2): 165-8. [Crossref]

28. Vargas-Ferreira F, Praetzel JR, Ardenghi TM. Prevalence of tooth erosion and associated factors in II-14-year-old Brazilian schoolchildren. J Public Health Dent 20II; 7I(I): 6-12. [Crossref]

29. Gurgel CV, Rios D, Buzalaf MAR, da Silva SMB, Araújo JJ, Pauletto $A G C$, et al. Dental erosion in a group of 12-and I6-year-old Brazilian schoolchildren. Pediatr Dent 20ll; 33(I): 23-8. [Crossref]

30. Auad SM, Waterhouse PJ, Nunn JH, Steen N, Moynihan PJ. Dental erosion amongst 13 -and 14 -year-old Brazilian schoolchildren. Int Dent J 2007; 57(3): 161-7. [Crossref] 